\title{
INSTRUMENTOS Y PROCEDIMIENTOS EN EVALUACIÓN NEURO- PSICOLÓGICA EN NIÑOS ONCOLÓGICOS, CON RIESGO DE DESARROLLAR DEFICITS COGNITIVOS
}

\section{INSTRUMENTS AND PROCEDURES IN NEUROPSYCHOLOGICAL ASSESSMENT OF CHILDREN WITH CANCER, WITH RISK TO DEVELOP COGNITIVE DEFICITS}

\author{
Águeda Sapiñal, ${ }^{1,}$ Tina Barahona ${ }^{2,3}$, Adela Cañete $^{2}$, Victoria Castel ${ }^{2}$, Jordi Bernabeu ${ }^{1,2}$ \\ 1 Unidad de Daño Cerebral Infantil. Hermanas Hospitalarias, Valencia. \\ 2 Unidad de Oncología Infantil. Hospital Universitario La FE, Valencia. \\ 3 Junta Asociada de Valencia de la Asociación Española Contra el Cancer
}

Resumen

En los últimos años el número de supervivientes del cáncer infantil ha aumentado considerablemente, esto ha permitido conocer las secuelas derivadas de la enfermedad y de sus tratamientos.

Diversos estudios han demostrado como la afectación de la sustancia blanca y gris por radioterapia y quimioterapia, y la localización del tumor, provocan diversas secuelas cognitivas y conductuales en estos niños.

El objetivo del presente trabajo pretende dar a conocer los instrumentos y procedimientos más utilizados en la exploración neuropsicológica infantil.

Puesto que en los niños los déficit pueden no aparecer hasta pasados varios años, planteamos la necesidad de realizar estudios neuropsicológicos amplios y de seguimiento que permitan conocer estas secuelas tanto a corto, medio y largo plazo.

Palabras clave: Neuropsicologia pediátrica, radioterapia, oncología pediátrica, efectos a largo plazo.

\section{Abstract}

Among the last years the survivor's number of childhood cancer has increased considerably, this has allowed to know the consequences resulting from the disease and its treatments.

Several studies have prooved how the involvement of white and gray matter radiotherapy and chemotherapy, and tumor location, lead to various cognitive and behavioral sequelae in these children.

The aim of this paper seeks to highlight the instruments and procedures used in the neuropsychological examination of children.

Because deficits in children may not appear until several years, that increases the need for extensive neuropsychological studies and monitoring that reveal these consequences in short, medium and long term.

Key words: Pediatric neuropsychology, radiotherapy, pediatric oncology, late effects.

\section{Correspondencia:}

Águeda Sapiña González

Unidad de Daño Cerebral Infantil Hermanas Hospitalarias

Eugenia Viñes, 15646011 Valencia

E-mail: asapina@hospitalariasmadrid.org 


\section{INTRODUCCIÓN}

Durante los últimos años el número de supervivientes del cáncer infantil ha aumentado de manera considerable. Este aumento de la supervivencia ha permitido conocer la secuelas tanto físicas como cognitivas derivadas de la enfermedad y sus tratamientos, desconocidas antes por el elevado número de mortandad ${ }^{(1)}$.

A diferencia de la población adulta en los que se establece una pérdida de funciones, en los niños, el daño cerebral por enfermedades $y / 0$ tratamientos producen una interferencia en el desarrollo de las capacidades en comparación con su grupo de iguales, ya que su celebro todavía no ha completado su desarrollo ${ }^{(2)}$. Estos déficit puede mostrarse de manera parcial hasta que se complete el desarrollo del cerebro o no aparecer hasta pasados varios años.

\section{PLANTEAMIENTO DEL PROBLEMA}

Teniendo en cuenta las teorías de la plasticidad, el daño cerebral temprano, puede producir una organización cortical anómala y transferir la función a un tejido sano, sin embargo, diversos hallazgos sugieren que la transferencia no es universal ${ }^{3,}$ 4, 5). El cerebro del niño, por tanto, puede recuperarse mejor de daños focales, pero de daños generalizados el pronóstico es peor dado que aunque suelen preservar funciones básicas, la recuperación cognitiva no suele ser completa ${ }^{(6)}$.

Hoy en día, se dispone de evidencia suficiente sobre el declive intelectual producido por la enfermedad y/o tratamientos en funciones de las distintas variables clínicas e individuales. En el campo de la oncología pediátrica, diversos trabajos describen los efectos a largo plazo de la radioterapia y quimioterapia, sobretodo del metrotexato en el sistema nervioso central ${ }^{(7)}$. Numerosos estudios (Filley et al. 2001; Palmer et al. 2002; Reddick et al. 2003) refieren los efectos de estos tratamientos, tanto en la sustancia blanca (leucoencefalopatía, pérdida de volumen...) como en la sustancia gris (atrofia, calcificaciones, cambios vasculares, necrosis... $)^{(8,9,10)}$.

Otros trabajos ya referían con anterioridad, que las personas con lesiones producidas durante la infancia suelen mostrar menores puntuaciones en las pruebas manipulativas debido posiblemente a que existe una mayor sensibilidad de estas tareas a las lesiones cerebrales ${ }^{(11,12)}$.

Algunos estudios han ido mostrando como los cocientes intelectuales descienden a lo largo del tiempo después de la radioterapia. Las medias (100) caen hasta los 70-80, tres años después del tratamiento ${ }^{(13)}$. Uno de los modelos que explicarían el origen de las dificultades académicas en estos niños es el modelo de Reddick. Este modelo explica como el tratamiento de radioterapia, en función de la edad y el tiempo transcurrido provocan un daño en sustancia blanca que repercute de forma generalizada en la atención, en la memoria, en el cociente intelectual y como consecuencia, en el rendimiento escolar ${ }^{(8,14)}$.

Diversos estudios (Levisohn et al. 2000; Schmahmann et al. 1998) $)^{(15,16)}$ refieren que los niños con inteligencia normal que adquieren lesiones cerebelosas (principalmente tumores), dependiendo de la localización concreta de la lesión presentan diferentes secuelas cognitivas y/o conductuales. Un ejemplo de ello, es el síndrome cognitivo afectivo cerebeloso que se ha descrito en niño y que aparece tras la resección de tumores. Este síndrome se caracteriza por el déficit en funciones ejecutivas (planificación y secuenciación), funciones visoespaciales, dificultades en lenguaje, memoria verbal y regulación del afecto. Parte de estos déficit, podrían explicarse por la relación que se ha encontrado entre el vermis y zonas orbitofrontales y del cíngulo anterior, y los hemisferios del 
cerebelo con zonas dorsolaterales y dorsomediales del córtex prefrontal ${ }^{(15,17)}$.

Así pues, aunque la evaluación neuropsicológica no establece la naturaleza, localización o extensión de una lesión subyacente, si puede determinar los déficit sensoriales, motores y cognitivos que de ella se derivan ${ }^{(2)}$.

\section{PROCESO DE EVALUACIÓN NEUROPSICOLÓGICA}

La variabilidad de los procedimientos empleados en la exploración neuropsicológica dependerá básicamente de la edad del niño y de su grado de desarrollo. A partir de los 7 años, la evaluación de un niño puede tener la misma extensión que la de un paciente adulto pero con criterios de corrección por edad. Los niños que presentan un menor grado de desarrollo pueden no ser capaces de realizar algunas pruebas estándar, en estos casos es recomendable centrarnos en establecer un nivel general de maduración y adaptación ${ }^{(5)}$. Para ello, una de las pruebas que proponemos es la escala de Comportamiento Adaptativo Vineland - II (18), que evalúa el comportamiento adaptativo en cinco dominios: Comunicación (lenguaje expresivo, lenguaje receptivo y lectura/escritura), Habilidades de la vida diaria (cuidado del hogar, cuidado de si mismo, vida en comunidad), Socialización (relaciones interpersonales, juego, ocio y tiempo libre $y$ adaptación), Habilidades motoras (fina y gruesa) y comportamiento adaptativo. Esta escala nos proporciona edades equivalentes aproximadas en cada una de las áreas y subáreas evaluadas.

Si la edad del niño y su grado de desarrollo lo permiten, la evaluación neuropsicológica debe incorporar medidas sensibles al funcionamiento del hemisferio derecho e izquierdo, ya que pueden verse alteradas las funciones de forma diferente $\mathrm{e}^{(14,19)}$.
Así pues, es importante realizar estudios de seguimiento que nos permitan conocer el impacto del cáncer y sus tratamientos, ya que como se ha mencionado anteriormente, en los niños el daño en el SNC puede no ser estático sino interrumpir los procesos de maduración y retrasar la aparición de los déficit ${ }^{(14)}$. Esto sugiere, que la exploración neuropsicológica debe realizarse de forma rutinaria, al menos en aquellos niños que han recibido irradiación craneal y que tenían menos de 8 años en el momento del diagnóstico ${ }^{(20)}$.

Por tanto, el procedimiento de evaluación neuropsicológico infantil debe estar compuesto por la exploración, el diagnóstico y el plan de intervención. De esta manera, la exploración lleva al diagnostico y éste guía el plan de acción. En pediatría es necesario que la evaluación englobe tres aspectos fundamentales: anamnesis, observación directa del niño y aplicación de pruebas ${ }^{(12)}$.

\section{Anamnesis}

En la entrevista inicial recogemos información relevante y esencial sobre todo el periodo comprendido entre el embarazo de la madre hasta la situación actual de enfermedad. Con ello obtenemos información de cómo ha sido el desarrollo del niño (motor, del lenguaje, socialización, conducta) durante todos los años de su vida y con anterioridad al momento del diagnostico, además de conocer los cambios que han podido aparecer por enfermedad y/o tratamientos y que pueden estar afectando a su día a día. Es importante además, tener en cuenta los posibles antecedentes de los familiares más cercanos (padres, tíos, abuelos...) que tengan relación con la patología del niño.

\section{Observación directa}

La observación del niño durante la realización de las pruebas es tanto o más 
importante que los resultados de las mismas, ya que es en ese momento, cuando podemos observar las estrategias que utiliza el paciente para realizar las tareas que se le piden, su comportamiento ante estas tareas, el lenguaje y habla, dificultades sensoriales, problemas de atención, habilidades motoras finas, interacción con los padres o con el terapeuta...

\section{Aplicación de pruebas}

Así pues, tal y como se propone en algunos trabajos en edad escolar y sobre todo en el campo de la oncología, el protocolo de evaluación debe ser extenso y estar compuesto de pruebas de habilidades generales, pruebas específicas y pruebas de habilidades académica ${ }^{(5,14,21)}$. Además, no podemos olvidar las dificultades conductuales y/o emocionales observadas en estos niños y la importancia por tanto, de incluirse pruebas psicopatológicas en el protocolo de la evaluación neuropsicológica.

\section{Pruebas de habilidades generales}

Las pruebas de habilidades generales son necesarias para poder establecer un cociente intelectual. Las más utilizadas son las escalas de inteligencia Wechsler para niños en sus dos versiones según la edad: la escala WPPSI-III (22), está indicada en edades comprendidas entre 2,5 años y 7,3 años, y está compuesta por índices sobre el funcionamiento intelectual en las áreas cognitivas verbal (Cl Verbal) y manipulativa (Cl Manipulativo), así como el índice $\mathrm{Cl}$ Total que representa la capacidad intelectual general del niño. La escala WISC$I^{(23)}$, se aplica a niños de entre 6 y 16 años y consta de 5 índices que miden el funcionamiento intelectual: comprensión verbal (CV), organización perceptiva (OP), memoria de trabajo (MT), velocidad de procesamiento (VP) y cociente intelectual total (CIT). Estas escalas, aunque no son pruebas neuropsicológicas, ofrecen información muy relevante para el diagnóstico.

Otra de las escalas utilizadas en edad escolar es la Batería de evaluación de Kaufman para niños $K-A B C^{(24)}$, es una prueba basada en la neuropsicología, psicología cognitiva y en estudios sobre la especialización cerebral, se aplica a niños de entre 2,5 y 12,5 años y evalúa aspectos cognitivos generales. Estima cocientes intelectuales y nivel de conocimientos. Con esta prueba se obtienen diversos factores globales: El procesamiento secuencial consta de 3 subpruebas relacionadas con tareas escolares diarias (decodificación lectora, memorización de hechos, comprensión de reglas del juego) y están asociadas a funciones de hemisferio izquierdo. El procesamiento simultáneo compuesto por 7 subpruebas relacionadas con la producción de soluciones que requieren la integración de la información y están asociadas a funciones de hemisferio derecho. El procesamiento mental compuesto que se obtendría con las puntuaciones de los dos factores anteriores y nos daría el nivel global de inteligencia. La escala de conocimientos, con la que se obtiene una medida del rendimiento y hechos adquiridos. Y por último, la escala no verbal que es aplicable a niños con dificultades en la comunicación oral y consta de subpruebas que requieren mímica y respuestas motoras.

\section{Pruebas específicas}

Las pruebas especificas, permiten hacer un barrido a todas las funciones cognitivas de manera detallada. Estas pruebas deben incluir las siguientes funciones: motricidad (rapidez motora ambas manos, coordinación visomotora), habilidades no verbales (praxias visoconstructivas, orientación de líneas, reconocimiento facial), lenguaje (vocabulario receptivo, comprensión gramatical...), memoria verbal inmediata, 
Tabla 1. Pruebas específicas

\begin{tabular}{|c|c|c|}
\hline PRUEBAS ESPECIFICAS & MEDICIÓN & EDAD APLICACIÓN \\
\hline $\begin{array}{l}\text { Continuos Performance Test II C. Keith } \\
\text { Conners (Conners' CPT-II / K-CPT) }{ }^{25,26)}\end{array}$ & $\begin{array}{l}\text { Mide indicadores de déficit de aten- } \\
\text { ción, impulsividad y vigilancia }\end{array}$ & $\begin{array}{l}\text { A partir de } 6 \text { años (CPT-II) } / 4-5 \text { años } \\
\text { (K-CPT) }\end{array}$ \\
\hline Grooved Pegboard ${ }^{(27)}$ & $\begin{array}{l}\text { Rapidez y la precisión motora en } \\
\text { cada mano }\end{array}$ & Desde los 5 años \\
\hline Peabody Picture Vocabulary Test-III (28) & Vocabulario receptivo & A partir de los 2 años y medio \\
\hline Token test for children ${ }^{(29)}$ & $\begin{array}{l}\text { Comprensión verbal con complejidad } \\
\text { y abstracción gramatical creciente }\end{array}$ & Puede usarse a partir de los 3 años. \\
\hline Benton Visual Retention Test ${ }^{(30)}$ & Coordinación visomotora & $\begin{array}{l}\text { Se puede utilizar con niños de a partir } \\
8 \text { años. }\end{array}$ \\
\hline Judgment of line orientation ${ }^{(31)}$ & Habilidad perceptiva visoespacial & A partir de los 7 años \\
\hline Figura compleja de Rey ${ }^{(32)}$ & Visoconstrucción & A partir de los 5 años \\
\hline $\begin{array}{l}\text { California Verbal Learning Test CA- } \\
\text { VLT-2 }\end{array}$ & $\begin{array}{l}\text { Aprendizaje serial de palabras y re- } \\
\text { cuerdo verbal demorado. }\end{array}$ & Desde los 5 hasta los 16 años. \\
\hline Stroop Color Word Test ${ }^{(34)}$ & $\begin{array}{l}\text { Capacidad de resistir la distracción e } \\
\text { inhibir la respuesta automática en la } \\
\text { lectura. }\end{array}$ & A partir de 7 años \\
\hline Childrens Color Trails Test ${ }^{(35)}$ & Función ejecutiva (Flexibilidad) & A partir de los 8 años \\
\hline $\begin{array}{l}\text { TOMAL, Test de Memoria y Aprendi- } \\
\text { zaje }^{(36)}\end{array}$ & $\begin{array}{l}\text { Memoria y el aprendizaje de tareas } \\
\text { verbales, no verbales y espaciales }\end{array}$ & De entre 5 y 19 años. \\
\hline $\begin{array}{l}\text { Behavior Rating Inventory of Executi- } \\
\text { ve Function (BRIEF) }\end{array}$ & $\begin{array}{l}\text { Mide habilidades de resolución de } \\
\text { problemas y auto-control a través de } \\
\text { la medida de } 8 \text { aspectos de funciona- } \\
\text { miento ejecutivo: Inhibición, flexibi- } \\
\text { lidad, control emocional, iniciativa, } \\
\text { memoria de trabajo, organización/ } \\
\text { planificación, organización de mate- } \\
\text { riales y monitorización }\end{array}$ & $\begin{array}{l}\text { De } 1,5 \text { años a } 5 . \\
\text { De } 5 \text { a } 18 \text { años. } \\
\text { De } 18 \text { a } 59 \text { años }\end{array}$ \\
\hline
\end{tabular}

aprendizaje y recuerdo verbal y visual, atención (sostenida, selectiva, dividida y alternante) y funciones ejecutivas (planificación, flexibilidad, secuenciación, interferencia). Algunas de las pruebas más utilizadas en función de la edad del niño, se describen a continuación (tabla 1)

\section{Pruebas de habilidades académicas}

Puesto que nuestros pacientes presentan edad escolar, es necesario incluir en la evaluación neuropsicológica pruebas de habilidades académicas. Estas deben darnos información sobre la lectura (decodi- ficación y comprensión), la escritura y la aritmética (cálculo y problemas). Para ello, algunas de las pruebas que podemos utilizar son subpruebas de la Batería $K-A B C^{(24)}$ (lectura/decodificación y lectura/comprensión, aritmética), de la Batería III de Woodcock-Muñoz (fluidez de la escritura, ortografía, identificación de letras y palabras, comprensión de textos, cálculo...) ${ }^{(38)}$ o de las escalas Weschler (aritmética ${ }^{(23)}$.

\section{Pruebas de conducta}

Como ya se ha comentado anteriormente, otro de los aspectos que debemos tener 
en cuenta son los problemas conductuales y/o emocionales. Los niños con afectación en el SNC pueden presentar dificultades en la regulación de conductas y/o emociones, por ello la evaluación neuropsicológica debe incluir entrevistas, registros y escalas de conducta tanto a padres como a profesores como por ejemplo el inventario de comportamiento $\mathrm{CBCL}^{(39-41)}$, en sus distintos formatos según edad e informantes, que nos den información detallada de las alteraciones conductuales/emocionales derivadas de la enfermedad y/o tratamientos.

\section{CONCLUSION/DISCUSIÓN}

Los niños con tumores cerebrales o leucemias que precisan de tratamiento directo sobre el sistema nervioso presentan, en muchos casos, afectación en el desarrollo del cerebro. Estas alteraciones, en ocasiones, pueden producir secuelas leves y no ser detectadas hasta pasados varios años después provocando con ello dificultades en diversos ámbitos de la vida (escolar, conductual, social...).

Por tanto, un extenso protocolo de valoración que abarque tanto las funciones cognitivas como los aspectos conductuales y/o emocionales y que nos permita seguir los déficit a largo plazo, es indispensable a la hora de establecer el plan de rehabilitación. Con ella obtendremos tanto los puntos fuertes de paciente como aquellas áreas o capacidades preservadas con el objetivo fundamental de planificar dicha intervención.

Si bien es cierto, debemos tener presente que estos pacientes no solo requieren de exploraciones neuropsicológicas, sino que necesitan además, de evaluación inicial, tratamiento y seguimiento desde un equipo interdisciplinar de profesionales, de manera que se evalúen y se traten todos los déficit que puedan ir surgiendo años después de la enfermedad y sus tratamientos.

\section{AGRADECIMIENTOS:}

Agradecimiento a Jordi Bernabeu Verdú, mi maestro y amigo, con el que he podido aprender y trabajar durante todos estos años. Jordi disfrutaba con su trabajo con los niños, enseñando en la facultad, investigando sobre todo en el campo de la oncología y daño cerebral adquirido infantil y eso, contagiaba a cualquiera que estuviera cerca. Aunque ya no está con nosotros, estoy segura que será el modelo a seguir por muchos profesionales de la neuropsicología, porque ha dejado una huella imborrable en la memoria de todos. GRACIAS JORDI. Águeda SG.

\section{REFERENCIAS BIBLIOGRÁFICAS}

1. Mostow EN, Byrne J, Connelly RR, Mulvihill JJ. Quality of life in long-term survivors of CNS tumors of childhood and adolescence. J Clin Oncol 1991;9: 592-9.

2. Sattler JM. Evaluación infantil. $3^{\circ}$ edición. Ed. Manual Moderno.1996. Mexico.

3. Anderson V, Catroppa C, Morse S, Haritou F, Rosenfeld J. Recovery of intellectual ability following TBI in childhood: impact of injury severity and age at injury. Pediatric Neurosurgery 2000; 32:382-90. Doi:10.1159/000028956

4. Anderson V, Godber T, Smibert E, Weiskop $\mathrm{S}$, Ekert $\mathrm{H}$. Impairments of attention following treatment whith cranial irradiation and chemotherapy in young children. $\mathrm{Br}$ J Cancer 2000; 82: 255-66.

5. Fournier C, Bernabeu J. Neuropsicología del daño cerebral adquirido infantil: evaluación y rehabilitación. Taller FEDACE sobre daño cerebral en niño y adolescentes, 2008; 7: 73-100.

6. Kolb B. Brain plasticity and behavior. Hillsdale, NJ: Lawrence Erlbaum Associates, Inc., 1995.

7. Mulhern RK, Khan RB, Kaplan S, Helton S, Chistensen R, Bonner M, et al. Shortterm efficacy of methylphenidate: A rand- 
omized, doublé-blind, placebo-controlled trial among survivors of childhood cancer. J Clin Oncol 2004; 22(23):4795-803. Doi: 10.1200/JCO.2004.04.128

8. Reddick WE, White HA, Glass JO, Wheeler GC, Thompson SJ, Gajjar A, et al. Developmental model relating white matter volume to neurocognitive deficits in pediatric brain tumor survivors. Cancer 2003; 97: 2512-9. Doi:10.1002/cncr.11355

9. Filley $\mathrm{CM}$, Kleinschmidt-DeMasters BK. Toxic Leukoencephalopathy. N Engl J Med 2001; 345:425-32. Doi:10.1056/ NEJM200108093450606

10. Palmer SL, Reddick WE, Glass JO, Gajjar A, Goloubeva O, Mulhern RK. Decline in corpus callosum volume among pediatric patients with medulloblastoma: Longitudinal MR imaging study. Am J Neuroradiol 2002; 23 (7):1088-94

11. Telzrow CF. Neuropsycological applications of common educational and psycological test. In: Reynolds CR, FletcherJanzen E, editors. Handbook of clinical child neuropsychology. New York: Plenum, 1989. p. 227-45

12. Manga D, Fournier C. Neuropsicología infantil. Estudio de casos en edad escolar. Editorial Universitas, SA, 1997.

13. Ris MD, Packer R, Goldwein J, Jones-Wallace D, Boyett JM. Intellectual outcome after reduced-dose radiation therapy plus adjuvant chemotherapy for medulloblastoma: a Children's Cancer Group study. J Clin Oncol 2001; 19 (15): 3470-6.

14. Bernabeu J, Cañete A, Fournier C, LopezLuengo B, Barahona T, Grau C, et al. Evaluación y rehabilitación neuropsicológica en oncología pediátrica. Psicooncología. 2003; 0 (1): 117-34.

15. Schmahmann JD, Sherman JC. The cerebellar cognitive affective syndrome. Brain 1998, 121: 561-79. Doi:10.1093/ brain/121.4.561

16. Levisohn L, Cronin-GolombA, Schmahmann JD. Neuropsychological consequences of cerebellar tumour resection in children.
Cerebellar cognitve affective syndrome in a paediatric population. Brain 2000; 123: 1041-50. Doi:10.1093/brain/123.5.1041

17. Schmahmann JD. Disorders of the cerebellum: ataxia, dysmetria of thougt, and the cerebellar cognitive affective syndrome. J Neuropsychiatry Clin Neurosci, 2004; 16(3), 367-78. Doi:10.1176/appi.neuropsych.16.3.367

18. Sparrow SS, Balla D, Cicchetti D. Vineland Adaptive Behavior Scale-Second Edition. Circle Pines, MN: American Guidance Services, 2005.

19. Holmes-Bernstein J. Developmental neuropsychology assessment. En Yeates $\mathrm{KO}$, Ris MD, Taylor HG, editors. Pediatric neuropsychology: Research, theory and practice. New York; Guildford Press, 1999; p. 405-38.

20. Pizzo PA, Poplack DG, editors. Principles and practice of pediatric oncology 4th Ed. Philadelphia: Lippincott Williams \&Wilkins Publishers, 2001

21. Fournier del Castillo MC. Neuropsicología de las demencias infantiles: adrenoleucodistrofia ligada a X. En Ruano A, editor. Neuropsicología Infantil, 2003, 337-358. Madrid: Ed. Mafpre.

22. Wechsler D. Escala de Inteligencia de Wechsler para preescolar y primaria (WPPSI-III). Madrid: Tea Ediciones, 2009.

23. Wechsler D. Wechsler intelligence Scale for Children $4^{\circ}$ Ed. (WISC- IV). Madrid: TEA Ediciones, 2005.

24. Kaufman LS, Kaufman NL. Batería de Evaluación de Kaufman para niños (K-ABC). Madrid: TEA Ediciones, 1997.

25. Conners CK, MHS Staff. Conners' CPT-II: Continuos performance test-II. Toronto, ON: Multi-Health System (MHS), 2002

26. Conners CK, MHS Staff. Conners' Kiddie Continuos performance test (K-CPT). Toronto, ON: Multi-Health System (MHS), 2001.

27. Grooved pegboard (mod.32025). Lafayette Instrument IN 47903 USA, 2002.

28. Dunn LM, Dunn LM. Examiner's annual for the Peabody picture vocabulary test (3rd 
ed.) Circle Pines, MN: American Huidance service, 1997.

29. De Renzi E, Bignolo LA. The Token Test: A sensitive test to detect receptive disturbances in aphasics. Brain, 1962; 85, 66578. Doi:10.1093/brain/85.4.665.

30. Benton AL. Revised Visual Retention Test: Clinical and experimental applications (4th Ed.). New York: The psychological Corporation, 1974.

31. Benton AL, Varney NR, Hamsher $K$. Visuospatial judgment: A clinical test. Arch Neurol 1978; 35:364-7. Doi:10.1016/00283932(78)90049-0.

32. Rey, A. Test de Copia de la figura compleja. Madrid: TEA, 1987.

33. Talley JL. Children's auditory verbal learning test2. Professional manual. Odessa, FL: Psychological Assessment Resources; 1996.

34. Golden, C. J. Stroop Color and Word Test: Manual for clinical and experimental uses. Chicago. Stoetling, 1978.

35. D'Elia LE, Staz P, Uchiyama CL, White T. Color Trails Test, Odessa, FL: PAR, 1996.
36. Reynolds CR, Bigler ED. Test de Memoria y Aprendizaje. Manual de Aplicación. Madrid: Tea Ediciones, 2001.

37. Gioia GA. Isquith PK, Guy SC, Kenworthy, L. Behavior Rating Inventory of Executive Function. Odessa, FL. Psychological Assessment Resources, 2000.

38. Woodcock RW, Muñoz-Sandoval AF, McGrew KS, Mather N. Batería III Woodcock Muñoz, 2005.

39. Achenbach TM, Rescorla LA. Manual for ASEBA Preschool Forms \& Profiles. Burlington, VT: University of Vermont, Research Center for Children, Youth, \& Families, 2000.

40. Achenbach TM, Rescorla LA. Manual for ASEBA School-Age Forms \& Profiles. Burlington, VT: University of Vermont, Research Center for Children, Youth, \& Families, 2001.

41. Achenbach TM, Rescorla LA. Manual for ASEBA Adult Forms \& Profiles. Burlington, VT: University of Vermont, Research Center for Children, Youth, \& Families, 2003. 